\title{
Retrofit Self-Optimizing Control of Tennessee Eastman Process
}

\author{
Lingjian Ye*, Yi Cao ${ }^{\dagger}$, Xiaofeng Yuan ${ }^{\ddagger}$ and Zhihuan Song ${ }^{\ddagger}$ \\ *Ningbo Institute of Technology, Zhejiang University, 315100, Ningbo, China, Email: lingjian.ye@gmail.com \\ ${ }^{\dagger}$ School of Energy, Environment and Agrifood, Cranfield University, Bedford MK43 0AL, UK, Email: y.cao@cranfield.ac.uk \\ ${ }_{\ddagger}^{\ddagger}$ Department of Control Engineering, Zhejiang University, 310027, Hangzhou, China, Email: yxf80, songzhihuan@zju.edu.cn.
}

\begin{abstract}
This paper considers near-optimal operation of the Tennessee Eastman (TE) process by using a retrofit selfoptimizing control (SOC) approach. Motivated by the factor that most chemical plants in operation have already been equipped with a workable control system for regulatory control, we propose to improve the economic performance by controlling some selfoptimizing controlled variables $(\mathrm{CVs})$. Different from traditional SOC methods, the proposed retrofit SOC approach improves economic optimality of operation through newly added cascaded SOC loops, where carefully selected SOC CVs are maintained at constant by adjusting set-points of the existing regulatory control loops. To demonstrate the effectiveness of the retrofit SOC proposed, we adopted measurement combinations as the CVs for the TE process, so that the economic cost is further reduced comparing to existing studies where single measurements are controlled. The optimality of the designed control architecture is validated through both steady state analysis and dynamic simulations.
\end{abstract}

\section{INTRODUCTION}

The well-known Tennessee Eastman (TE) challenge problem was published by [1], based on an actual industrial process. The plant consists of the following 4 reactions

$$
\begin{aligned}
A(g)+C(g)+D(g) & \rightarrow G(l i q) \\
A(g)+C(g)+E(g) & \rightarrow H(l i q) \\
A(g)+E(g) & \rightarrow F(l i q) \\
3 D(g) & \rightarrow 2 F(l i q)
\end{aligned}
$$

where $A, C, D, E$ are the reactants, $G$ and $H$ are the desired products and $F$ is the byproduct. Besides, there exists an inert component $B$ in the material circle, which is contained in the feed and removed through the purge to maintain inventory balance. The process includes 5 major operating units: the reactor, a product condenser, a vapor-liquid separator, a recycle compressor and the product stripper.

Downs and Vogel [1] posed various control and optimization tasks to operate such a plant-wide process, e.g. (1) track the set-points of some key variables (e.g. production rate and product quality) quickly and smoothly with set-point changes and/or disturbances; (2) minimize the valve movements (control energy) along with set-point tracking; (3) optimize the economic cost for daily operation, etc. To deal with this TE challenge problem, systematic approaches for both control structure and controller design have been applied to the TE problem. McAvoy and Ye [2] configured a basic PID control system using the relative gain array and other controllability analysis tools, which met basic requirements posed in the problem. However, their control system was operated around the base case values provided by Downs and Vogel [1], which is economically expansive. To improve the economic performance, Ricker [3] solved out optimal steady state working points for all 6 defined operating modes. It was demonstrated that compared to the base case, a significant cost reduction (about $1 / 3$ ) can be realized by operating the optimal point identified. He also presented a well-configured decentralized control structure [4], which achieved excellent performances for various control tasks. In other works, model predictive control was also considered to complete the posed control tasks for the TE process [5], [6]. Larsson et al. [7] applied the self-optimizing control (SOC) strategy [8] to improve the economic optimality via selecting controlled variables $(\mathrm{CVs})$ for the control system. Besides of the conventional constrained variables (e.g. liquid levels, production rate and quality), they selected the reactor temperature, recycle flowrate and the mole fraction of $C$ in the purge as the CVs, so that the operation is self-optimizing under disturbances and different operating conditions. Also, they showed that the improvements were mainly achieved in the case of production rate changes, as compared to the one in [4].

It is well known that adopting measurement combinations as $\mathrm{CVs}$ is better than using single measurements. Many approaches have also been developed for measurement combination CVs [9], [10], [11], [12], [13]. Nevertheless, an application of measurement combination CVs to the well-known TE process has not yet been reported elsewhere. The reason may be due to the inherent control difficulty of the TE process, which is open-loop unstable and highly integrated. This inherent control difficulty makes adopting measurement combination CVs complicated if the SOC loops have to be designed together with the basic regulatory control. This complexity is alleviated through the proposed retrofit approach, where the SOC is implemented in an upper cascade layer so that the inherent regulatory control difficulty is independent from the SOC design. Another reason is perhaps that most SOC methods in the literature were developed under the assumption that uncertainties the system suffered can be well described by a few "disturbance variables". Moreover, the operation is perturbed around a nominal point when these disturbance vari- 
ables change. However, the TE process is operated with several particular disturbance scenarios and operating conditions, it is difficult to define some consistent "disturbance variables", which should be known to the designers for most existing SOC methods. Recently, we have developed a new SOC approach for CV selection [14], which approximately minimizes the average economic loss of all possible "disturbance scenarios" or more generally say, "operating conditions", hence wellsuited for the TE background.

The remainder of this paper is arranged as follows: Section 2 introduces some backgrounds of the control problem for the TE process, two existing control structures of interest [4], [8] are mainly reviewed. In Section 3, we briefly outline a newly developed SOC approach, which minimizes the global average loss for all operating conditions and well-suited for addressing the TE problem. Results for self-optimizing CVs and simulations are provided in Section 4. Finally, Section 5 concludes this paper.

\section{CONTROL PROBLEM FOR TE PROCESS}

The TE process includes 12 manipulated variables (MVs) and 41 measurements [1]. For the MVs, they have all been scaled within 0-100 limits in percentage as provided in the benchmark problem. For the measurements, they are defined with different sampling frequencies and dead time to keep consistence with the industrial practice, see [1] for more details.

Although many control structures have been developed for the TE process in the literature, in the remainder of this paper, we will focus on the ones proposed by [4] and [7] due to their efficiency in performance. (For the sake of convenience, they will be denoted as "CS_Ricker" and "CS_Skoge" afterwards.) Furthermore, the choice of $\mathrm{CV}$ selection is of particular interest in this study and reviewed below. According to their control policies, the following process variables should be controlled in closed-loops:

1) Separator level and stripper level. These two liquid levels are integrating variables and have no steady state effects, they must be stabilized in the first place.

2) Production rate (stripper underflow) and product quality (mole $\% G$ in product). Manufacturing objective defines their targets under different operating modes and specifications, these constitute equality constraints and should be controlled to satisfy the targets. (Except that for mode 4-6, the production rate is aimed to be maximized.)

3) At the optimum, there are 5 active constraints that needs to be controlled at their boundaries: reactor pressure (maximum) and level (minimum), compressor recycle valve (closed), stripper steam valve (closed) and agitator speed (maximum). [4] provided detailed physical interpretations why these constraints are active at the optimum.

Above control requirements consume 9 degrees of freedom (DOF) for plant operation. For the remaining unconstrained 3 DOF, [4] chose to control the reactor temperature $\left(T_{r c t}\right), \% A$ and $\% C$ in the feed (more precisely, $y_{A}$ : the combined $\% A+$
$\% C$ and $y_{A C}: \% A /(\% A+\% C)$ in the feed $)$ based on heuristic analysis. Decentralized control structure was considered with appropriately configured loop pairing relationships. Controller tuning is also carried out for all PI controllers, see [4] for more detail. The designed control system was very efficient and nicely completed various control tasks proposed by [1] .

On the other hand, [7] applied the so-called SOC methodology to improve the operational economic performance for TE process. Their control strategy addressed the situations when the plant is operated under disturbances and different operating conditions (specifically, production rate/throughput change by $\pm 15 \%)$. A systematic procedure for control structure design and self-optimizing CVs selection was carried out. They found that the most promising $\mathrm{CVs}$ for the remaining unconstrained 3 DOF are reactor temperature $T_{r c t}$, recycle flowrate $\left(F_{\text {recycle }}\right)$ and $\% C$ in the purge $\left(y_{C_{\text {purge }}}\right)$. These results were at first surprising and contradicting to engineers' insights of process control, however, the authors had conducted dynamic simulations to demonstrate that their control strategy is viable. As compared to the one in [4], the main economic improvements were achieved in the cases of throughput changes.

\section{A RETROFit SOC METHODOLOGY WITH AN ENHANCED CV SELECTION METHOD}

\section{A. A retrofit SOC methodology}

The current industrial practice is that for existing chemical plants, they have already been installed with control systems working reasonably well in terms of important control tasks, although they may not be necessarily optimal from an economic perspective. Practically, designers advocating SOC methodology face the following challenges:

1) Develop an efficient SOC method for CV selection, which may necessarily be a multi-objective task, e.g. it has been realized that one should seek a compromise between the economic performance and CV complexity.

2) Systematic solution to improve economic performance on basis of an existing control system without redesigning a fresh one. This way, one is allowed to pay minimal efforts and preserve important merits of an existing control system.

3) Convince the man in charge of plant operation to commission the designed self-optimizing control system.

Up to date, the first issue is intensively studied by researchers in this area and a number of outcomes have been reported. However, the others are highly relevant to industrial practice and less discussed. To the best experiences of the authors, the field operators/engineering would rather rely on their in-position control systems because they commonly control single measurements thus easily fit into peoples' physical judgements. On the other hand, a well configured SOC system often uses measurement combinations as $\mathrm{CVs}$, which lacks clear physical interpretations. In this context, it is hardly possible to persuade chemical factories risk shutting down current operation and then installing a possibly deleterious control system (in their minds). 
Above mentioned truths necessitate the development of an SOC methodology compatible to existing control systems, which are mainly designed for regulatory control purpose. Two requirements should be met in order: (1) Fundamental regulatory control tasks should be satisfied. In any circumstance, there should exist an interface that allows one to restore back into the former control system easily. This helps increase the operators' confidence on the new control system. (2) Economic performance is improved by controlling the self-optimizing $\mathrm{CV}$ s obtained. In this paper, we propose a so-called retrofit SOC methodology to address these requirement. Although the concept of retrofit is widely adopted for process improvement (e.g. [15] and refs therein), it is the first time to apply such a concept to implement SOC. Existing methodologies in the literature only consider design SOC structure in the blueprint phase, however, the retrofit SOC proposed in this work shows a way in which SOC can be implemented in operational processes.

Suppose that there exists an in-position control system (regulatory control) in usage, the $\mathrm{CVs}$ are firstly divided into two classes: (1) $y_{1}$, including variables that have no steady state effects (e.g. liquid levels) and active constraints (equality variables); (2) $y_{2}$ : other CVs except for $y_{1}$. As we know, $y_{2}$ should be replaced with self-optimizing CVs for a better economic performance, as in a tradition SOC framework. However, in the proposed retrofit SOC methodology, we configure an independent control layer (self-optimizing control layer) upon the in-position control system. In the upper self-optimizing control layer, the obtained CVs for SOC are controlled by adjusting the set-points of $y_{2}$, which are further sent downwards to the lower regulatory control layer and be tracked. The proposed control system has the following features:

- Regulatory control tasks are naturally addressed by the preserved control system in lower layer;

- By controlling the self-optimizing CVs in upper layer, the economic performance of daily operation is improved;

- Interfaces are left to interact with the new control system. For example, since controlling measurement combinations are not easily understood by the field operators, a saturation block can be imposed on the controller outputs in the upper layer by choosing a reasonable range of $y_{2}$ 's setpoint, which makes them feel safe and confident. Furthermore, it is also convenient to restore back to former control system, simply by switching the controllers in upper layer into "manu" mode. When operated in the "auto" mode, it would be agreeable to adjust set-points of $y_{2}$, which can be constrained in safe ranges.

\section{B. A new SOC method for CV selection}

Recently, the authors of this paper have developed a new SOC approach for CV selection [14], which approximately minimizes the average loss under all operating conditions. Although two algorithms are proposed therein, in the sequence, the less rigourous one is presented, which has an appealing advantage that the CVs are solved analytically based on the optimal values of measurements obtained. Furthermore, the sensitivity matrix required in the algorithm is allowed to be evaluated at a single reference point.

Consider the next static optimization problem

$$
\min _{\mathbf{u}} J(\mathbf{u}, \mathbf{d})
$$

with measurements

$$
\mathbf{y}=\mathbf{f}(\mathbf{u}, \mathbf{d})
$$

where $J$ is the cost function to be minimized, $\mathbf{u} \in \mathbb{R}^{n_{u}}, \mathbf{d} \in$ $\mathbb{R}^{n_{d}}$, and $\mathbf{y} \in \mathbb{R}^{n_{y}}$ are the manipulated variables, disturbances, and measurements, respectively. $\mathbf{f}: \mathbb{R}^{n_{u} \times n_{d}} \rightarrow \mathbb{R}^{n_{y}}$ is the measurement model. The objective is to select $\mathbf{c}=\mathbf{H y}$ as CVs such that the economic loss is minimized under different disturbance scenarios and operating conditions.

Remark: Although the symbol $\mathbf{d}$ is used above to denote disturbances [14], the method can be easily extended to any possible operating conditions that cannot be easily described by simple disturbance variables, e.g. a particular case with a pressure setpoint change or a case of instrument failure. Also, since occurrence of disturbance is considered as a type of operating condition, we will refer the term "operating condition" to all possibilities afterwards, without loss of generality.

A brief description of the algorithm [14] involves the following steps:

1) For all $N$ operating conditions, say $\mathbf{d}_{(i)}, i=1, \ldots N$, the cost function $J$ is minimized using an optimization solver. The optimal values of the measurements, $\mathbf{y}_{(i)}^{o p t}$ are stored to form a matrix as

$$
\mathbf{Y}=\left[\begin{array}{llll}
\left(\mathbf{y}_{(1)}^{\text {opt }}\right)^{\mathrm{T}} & \left(\mathbf{y}_{(2)}^{\text {opt }}\right)^{\mathrm{T}} & \cdots & \left(\mathbf{y}_{(N)}^{\text {opt }}\right)^{\mathrm{T}}
\end{array}\right]^{\mathrm{T}}
$$

Here, the measurement vector $\mathbf{y}$ is defined to include an artificial measurement: constant 1 , through which means the set-points of all final derived CVs are 0.

2) In the presence of measurement noises, construct an extended matrix $\tilde{\mathbf{Y}}$ as

$$
\tilde{\mathbf{Y}}=\left[\begin{array}{l}
\frac{1}{\sqrt{N}} \mathbf{Y} \\
\mathbf{W}_{n}
\end{array}\right]
$$

where $\mathbf{W}_{n}$ is a diagonal matrix with its diagonal elements as the error magnitudes of each measurement.

3) Choose a particular operating point as the reference point, the gain matrix of $\mathbf{y}$ with respect to the MVs is evaluated as $\mathbf{G}_{y, r e f}$.

4) Simply, the $\mathrm{CV}$ combination matrix $\mathbf{H}$ can be computed as $\mathbf{H}^{\mathrm{T}}=\left(\tilde{\mathbf{Y}}^{\mathrm{T}} \tilde{\mathbf{Y}}\right)^{-1} \mathbf{G}_{y, \text { ref }}$. (In a noise-free case, replace $\tilde{\mathbf{Y}}$ with $\mathbf{Y}$ ).

Note, solution for $\mathrm{CV}$ selection is basically not unique, provided that only steady state performance is concerned. A universal expression for the combination matrix should be $\tilde{\mathbf{H}}=\mathbf{B H}$, where $\mathbf{B}$ is any $n_{u} \times n_{u}$ nonsingular matrix. However, one favorable choice is selecting $\mathbf{B}=\left(\mathbf{H G}_{y, r e f}\right)^{-1}$ such that the gain from $\mathbf{u}$ to $\mathbf{c}$ is $\mathbf{I}$, which achieves decoupling control at the reference point for a multi-variable system thus facilitates dynamic control. 
TABLE I

OPTIMIZATION RESULTS

\begin{tabular}{llllc}
\hline & $\operatorname{Cost}[\$ / \mathrm{h}]$ & $y_{A}[\%]$ & $y_{C}[\%]$ & rct temp ${ }^{\circ} C$ \\
\hline normal & 114.01 & 32.21 & 18.75 & 122.9 \\
IDV(1) & 111.27 & 32.35 & 19.69 & 123.0 \\
IDV(2) & 169.03 & 30.47 & 17.94 & 124.2 \\
throughput $+15 \%$ & 140.55 & 33.45 & 19.68 & 124.3 \\
throughput $-15 \%$ & 91.01 & 30.80 & 17.50 & 121.6 \\
40 G/ 60 H & 129.07 & 32.92 & 18.93 & 123.4 \\
rct press $2645 \mathrm{kPa}$ & 134.93 & 32.01 & 18.82 & 123.6 \\
\hline
\end{tabular}

\section{SElF-OPTIMIZING CONTROL OF THE TE PROCESS}

In this section, the TE process is studied to pursue better economic performance, with application of the $\mathrm{CV}$ selection method and SOC methodology described above.

\section{A. CV selection}

First of all, the same $9 \mathrm{CVs}$, including two liquid levels with no steady-state effects and 7 equality constraints (see Section 2), are controlled. These CVs are selected with the same reasons as was done in CS_Ricker and CS_Skoge. Consequently, we are left with 3 unconstrained DOF for SOC purpose.

For operating conditions, [7] considered IDV(1) and IDV(2), because the other disturbances defined by [1], either have no steady state effects or too severe to be handled (IDV(6): loss of feed A, recommended to be handled with override control). Besides, they also considered the situations when the set-point of production rate (throughput) is changed by $\pm 15 \%$. Besides of these, we additionally incorporate two situations as posed by Downs and Vogel [1]: (1) when the product mix changes from $50 \mathrm{G} / 50 \mathrm{H}$ to $40 \mathrm{G} / 60 \mathrm{H}$; (2) a step change of set-point for reactor pressure to be $2645 \mathrm{kPa}$. Therefore, there will be 7 operating conditions in total (including the normal operating condition) investigated in this study.

The operating cost of TE process, $J$ is defined consisting of purge cost, product stream cost, compressor cost and steam cost. Firstly, optimization (minimizing $J$ ) for all 7 operating conditions are performed using a Genetic Algorithm. Since there are only 3 sensitivity-seeking DOF for optimization, the algorithm is based on CS_Ricker, where the set-points of $y_{A}$, $y_{A C}$ and $T_{r c t}$ are used as the decision variables to minimize $J$, i.e.

$$
\mathbf{u}=\left[\begin{array}{lll}
y_{A} & y_{A C} & T_{r c t}
\end{array}\right]^{T}
$$

in the formulated optimization problem.

Results of the minimal cost and optimal decision variables are summarized in Table 3. Meanwhile, optimal values of all 41 measurements are also obtained for these 7 cases (numerical values are not shown here).

The measurement subset for self-optimizing CV selection is chosen as

$$
\mathbf{y}=\left[\begin{array}{llllll}
1 & y_{A} & y_{A C} & T_{\text {rct }} & y_{C_{\text {purge }}} & F_{\text {recycle }}
\end{array}\right]^{T}
$$

which are controlled by either CS_Ricker or CS_Skoge. Finite difference method is used to evaluate the sensitivity matrix $\mathbf{G}_{y}$
TABLE II

ECONOMIC LOSSES [\$/H] OF 3 CONTROL SYSTEMS

\begin{tabular}{llll}
\hline & CS_Ricker & CS_Skoge & This paper \\
\hline normal & $\mathbf{0 . 0}$ & $\mathbf{0 . 0}$ & 0.03 \\
IDV(1) & $\mathbf{0 . 0}$ & 0.03 & 0.03 \\
IDV(2) & 2.7 & 1.7 & $\mathbf{1 . 6}$ \\
throughput $+15 \%$ & 6.1 & 1.5 & $\mathbf{0 . 0}$ \\
throughput $-15 \%$ & 2.6 & 0.6 & $\mathbf{0 . 0}$ \\
40 G/ 60 H & 0.7 & $\mathbf{0 . 3}$ & 0.9 \\
rct press 2645 kPa & 0.3 & 4.5 & $\mathbf{0 . 2}$ \\
\hline sum & 12.4 & 8.63 & 2.76 \\
\hline
\end{tabular}

, by perturbing the set-points of $y_{A}, y_{A C}$ and $T_{r c t}$ around the normal operating condition. $\mathbf{G}_{y}$ is obtained as

$$
\mathbf{G}_{y}=\left[\begin{array}{ccc}
0 & 0 & 0 \\
1 & 0 & 0 \\
0 & 1 & 0 \\
0 & 0 & 1 \\
0.0755 & 1.167 & -0.0395 \\
0.564 & 0.166 & -0.221
\end{array}\right]
$$

Then, the algorithm described in Section 3 is applied and the $\mathrm{CV}$ combination matrix $\mathbf{H}$ is solved as

$\mathbf{H}=\left[\begin{array}{cccccc}-505.9 & 23.75 & -10.79 & 1.38 & -28.82 & 4.60 \\ 973.4 & -51.59 & 20.02 & -1.80 & 66.12 & -10.08 \\ -170.9 & 1.35 & -4.11 & 1.49 & 1.29 & 0.10\end{array}\right]$

\section{B. Steady state evaluation}

The economic performance of obtained self-optimizing CVs are validated against CS_Ricker and CS_Skoge. The economic losses are computed by assuming that all CVs are perfectly controlled at the steady state for all the 3 investigated control systems, as listed in Table 4. CS_Skoge outperforms CS_Ricker mainly in the case of throughput changes, which verifies those results provided in their paper [7]. However, when the set-point of reactor pressure is set to be $2645 \mathrm{kPa}$, which has not considered by [7], CS_Skoge gives even a poorer economic loss of $4.5 \$ / \mathrm{h}$ than CS_Ricker $(0.3 \$ / \mathrm{h})$.

Improvements can be made by selecting measurement combinations as CVs, as validated in this paper. For the 7 investigated cases, smaller economic losses are achieved for those cases when either CS_Ricker or CS_Skoge give a large loss. For example, in the case of throughput $\pm 15 \%$, CS_Ricker gives a loss of $6.1 \$ / \mathrm{h}$ and $2.6 \$ / \mathrm{h}$, respectively. Whilst our approach gives both 0 losses. When the setpoint of reactor pressure changes, in which case CS_Ricker performs bad, our approach achieves a loss of $0.2 \$ / \mathrm{h}$. In the case of normal operating and product mix changes, our approach does not perform better, however, the sacrificed loss is very small. In summary, by controlling the measurement combinations, this paper achieves a total loss of $2.76 \$ / \mathrm{h}$ for the 7 investigated cases, which is substantially reduced as compared to CS_Ricker and CS_Skoge (12.4 \$/h and 8.63 $\$ / h)$.

\section{Dynamic simulations}

Since CS_Ricker was so well-configured in stabilizing the plant operation and completing fundamental control tasks, it 
will be used as a basis to control the obtained self-optimizing CVs. Following the proposed retrofit SOC methodology, an independent control layer (3-inputs-3-outputs) is built upon CS_Ricker. The 3 self-optimizing CVs are controlled by adjusting set-points of $y_{A}, y_{A C}$ and $T_{r c t}$, which are further sent to the lower control loops for set-point tracking. A decentralized control strategy is considered, to this end, a decoupling combination matrix $\tilde{\mathbf{H}}=\left(\mathbf{H G}_{y}\right)^{-1} \mathbf{H}$ is calculated as

$$
\tilde{\mathbf{H}}=\left[\begin{array}{cccccc}
-31.2 & 0.804 & -1.239 & 0.085 & 0.825 & 0.238 \\
-7.58 & -0.084 & -0.435 & 0.043 & 0.977 & 0.017 \\
-99.0 & -0.031 & -2.243 & 1.028 & 1.547 & -0.153
\end{array}\right]
$$

to enhance the controllability between the inputs and outputs. From a steady state point of view, the performances of $\mathbf{H}$ and $\tilde{\mathbf{H}}$ are equivalent. However, using $\tilde{\mathbf{H}}$ is easier for implementation because the inputs and outputs are decoupled. PI controllers are employed for all loops configured in the upper control layer.

For illustrative purpose, in the following, we firstly investigate an arranged series of operating scenario as: (1) Initially, the system is operated under normal condition; (2) At $10 \mathrm{~h}$, the setpoint of production rate (throughput) is set by $+15 \%$. To avoid abrupt fluctuation, the set-point change is ramped within a period of $10 \mathrm{~h}(10 \mathrm{~h}-20 \mathrm{~h})$; (3) At $80 \mathrm{~h}$, the production rate is reset to normal condition, simultaneously, the set-point of reactor pressure is changed from $2800 \mathrm{kPa}$ to $2645 \mathrm{kPa}$. Similarly, both the set-point changes are ramped within a period of $10 \mathrm{~h}$.

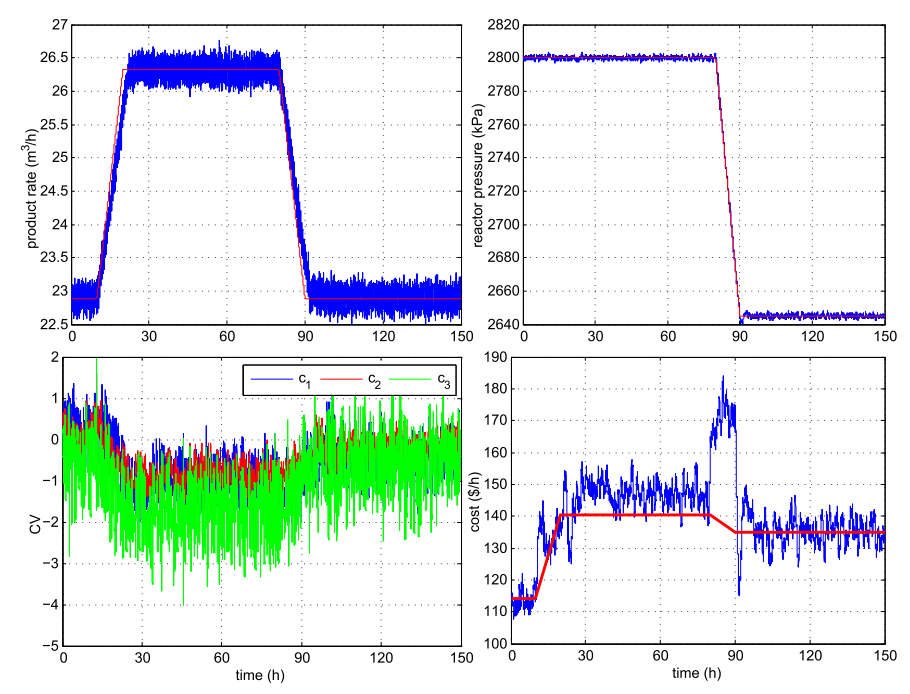

Fig. 1. Dynamic performance of CS_Ricker

As a comparison, CS_Ricker and CS_Skoge are both tested for the given operating scenarios, as shown in Figure 1 and 2. It is evident that both of them work well in tracking the specified set-point changes, for both the production rate and reactor pressure. However, their economic performances are somewhat different.
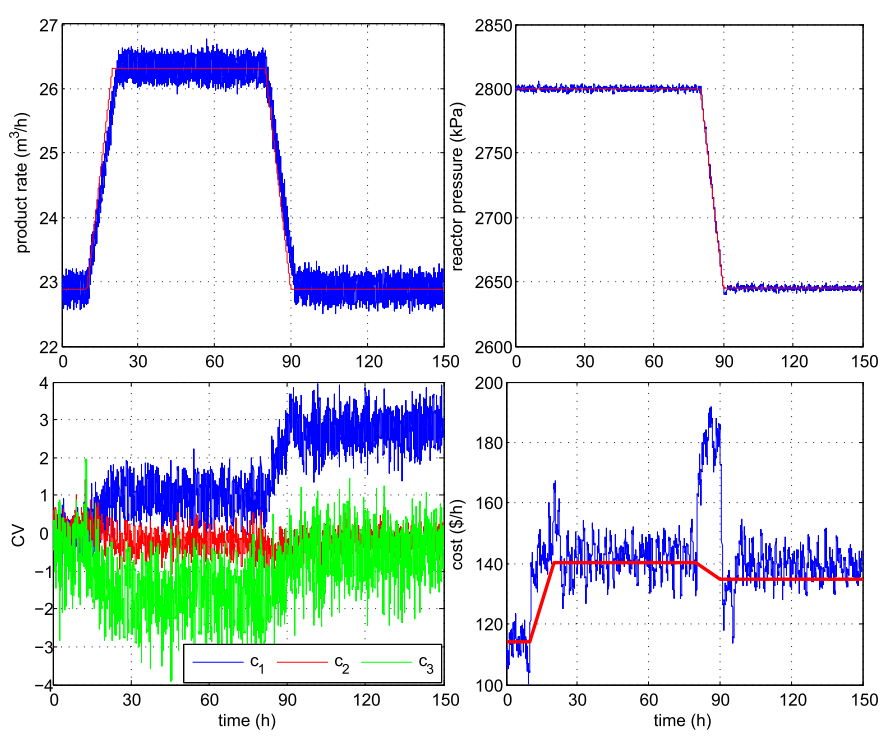

Fig. 2. Dynamic performance of CS_Skoge

To better explore the detail, we calculate the instantaneous value of the 3 self-optimizing $\mathrm{CVs}, \mathbf{c}=\mathbf{H y}$ in the whole simulation time. (Note that, for these two control systems, $c=$ Hy are not controlled hence they can be regarded as "open loop" systems.) It is interesting to note that, the $3 \mathrm{CVs}$ values in CS_Ricker all deviate from 0 significantly at about 20-80 h (throughput $+15 \%$ ). Meanwhile, the operating cost runs above the minimal cost line (140.55, red line) under this operating conation. However, when the reactor pressure is maintained at $2645 \mathrm{kPa}(90-150 \mathrm{~h})$, the $3 \mathrm{CVs}$ go back to near 0 and the operating cost is around the minimal cost (134.93). These dynamic economic performances are in agreement with those results obtained in steady state analysis, namely, CS_Ricker gives economic losses of 6.1 and 0.3 for the two investigated operating conditions, respectively. Finally, in the whole $150 \mathrm{~h}$ simulation time, the overall economic cost for CS_Ricker is calculated as 21088 \$.

For CS_Skoge, it is observed that during 20-80 h, $c_{1}$ and $c_{3}$ deviate away from 0 in certain extent whilst $c_{2}$ maintains near 0 . The economic cost, however, is maintained (virtually) around the minimal value, which indicates that the economic loss is not large. In the phase of 90-150 h, $c_{1}$ deviates largely from 0 while the other $2 \mathrm{CVs}$ are quite near 0 . Consequently, the economic cost approximately runs above minimal red line, thus a notable loss occurs. Again, the dynamic simulation results are in accordance with the steady state analysis, which gives losses of 1.7 and 4.5 respectively. The final operating cost for this control system is 21195 \$, which is even worse than CS_Ricker for the arranged operating conditions.

Finally, the proposed retrofit SOC control system is tested with the measurement combinations controlled. The results in Figure 3 indicates following points:

1) The specified setpoint changes for both production rate and reactor pressure are tracked quickly and smoothly, 

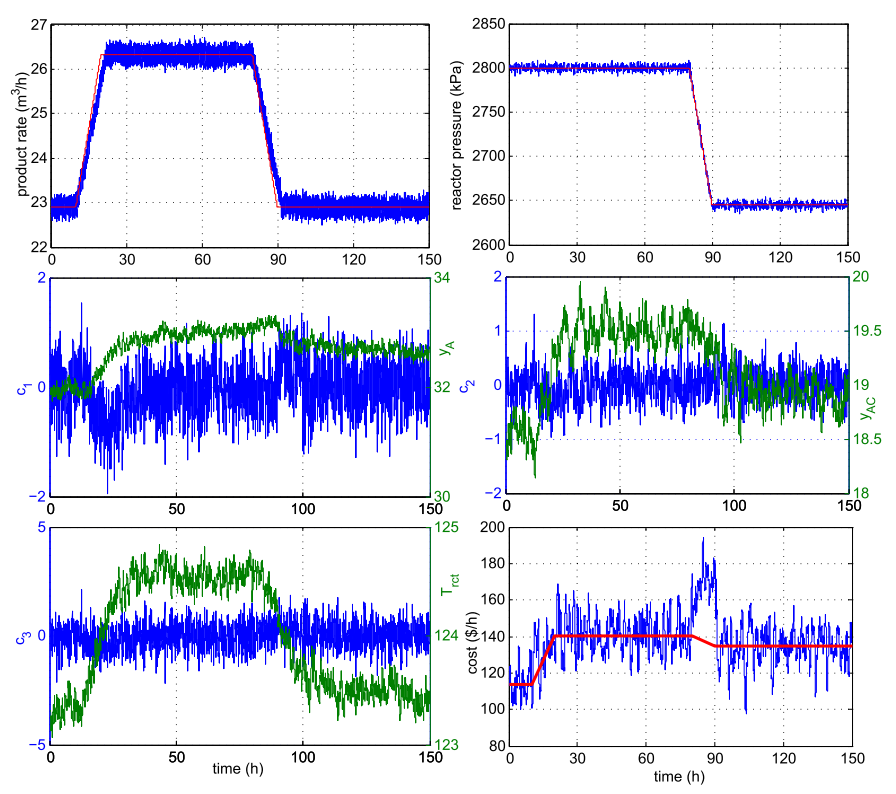

Fig. 3. Dynamic performance of controlling the control system in this paper

because the control system is directly configured upon CS_Ricker with the lower regulatory controllers unchanged, hence the merits of CS_Ricker are preserved.

2) In spite of operating condition switches, the 3 selfoptimizing CVs are controlled around 0 with the function of new added PI controllers, by adjusting the setpoints of $y_{A}, y_{A C}$ and $T_{r c t}$.

3) The economic cost is automatically operated around the minimum for the all simulation time, thus a good economic performance is attained. The overall operating cost in the $150 \mathrm{~h}$ duration is calculated as 20722 , which saved 366 \$ and 473 \$ as compared to CS_Ricker and CS_Skoge, respectively.

Besides of above simulations, all the 7 operating scenarios have also been simulated independently. Table III summarizes the average costs in an operation time of $150 \mathrm{~h}$, with the system states initially as steady state values under the normal condition. The verified dynamic performances are similar to steady state results. Among 7 operating conditions tested, the proposed one achieves minimal average cost for 4 cases (in the presence of measurement noises, we consider a difference of \pm 0.1 average loss is negligible), namely, normal condition, throughput $\pm 15 \%$, and reactor pressure at $2645 \mathrm{kPa}$. Furthermore, in the other remaining 3 cases, the differences between then best control system and the proposed one are also very small although it is not optimal. For example, in the case of IDV(2) the minimal average loss is 170.35 (CS_Skoge) whilst the one in this study is 170.75 . See Table III for more of such results.

\section{CONCLUSIONS}

A retrofit SOC control system is configured upon an existing control system (CS_Ricker) for the TE plant. In the
TABLE III

AVERAGE COST [\$/H] FOR 7 OPERATING CONDITIONS

\begin{tabular}{llll}
\hline & CS_Ricker & CS_Skoge & This paper \\
\hline normal & $\mathbf{1 1 4 . 0 0} \pm \mathbf{3 . 4 5} *$ & $\mathbf{1 1 3 . 9 4} \pm \mathbf{4 . 4 5}$ & $\mathbf{1 1 4 . 0 4} \pm \mathbf{8 . 1 7}$ \\
IDV(1) & $111.62 \pm 16.15$ & $\mathbf{1 1 1 . 4 9} \pm \mathbf{7 . 7 6}$ & $111.63 \pm 15.84$ \\
IDV(2) & $171.85 \pm 5.13$ & $\mathbf{1 7 0 . 3 5} \pm \mathbf{5 . 8 8}$ & $170.75 \pm 8.70$ \\
throughput $+15 \%$ & $147.15 \pm 21.69$ & $143.15 \pm 20.61$ & $\mathbf{1 4 1 . 5 4} \pm \mathbf{2 2 . 4 4}$ \\
throughput $-15 \%$ & $93.41 \pm 11.48$ & $\mathbf{9 0 . 3 5} \pm \mathbf{8 . 7 8}$ & $\mathbf{9 0 . 2 7} \pm \mathbf{1 1 . 8 1}$ \\
$40 \mathrm{G} / 60 \mathrm{H}$ & $130.85 \pm 25.76$ & $\mathbf{1 3 0 . 6 0} \pm \mathbf{2 0 . 1 7}$ & $131.23 \pm 26.78$ \\
rct press $2645 \mathrm{kPa}$ & $\mathbf{1 3 7 . 6 8} \pm \mathbf{3 0 . 0 7}$ & $142.89 \pm 35.02$ & $\mathbf{1 3 7 . 7 8} \pm \mathbf{3 0 . 6 8}$ \\
\hline \multicolumn{4}{c}{$a \pm b: a$ is the average loss and $b$ is the standard deviation }
\end{tabular}

independently configured SOC layer, the derived measurement combinations are controlled to achieve better self-optimizing performance, by adjusting set-points of $y_{A}, y_{A C}$ and $T_{r c t}$. The optimality of the designed control architecture is validated both through steady state analysis and dynamic simulations.

However, there are still some aspects remained unsolved in this paper, for example, self-optimizing control for other 5 operating modes for the TE process, and the measurement subset selection problem for constituting self-optimizing CVs. These challenges will be further investigated in an extension of this study.

\section{REFERENCES}

[1] J. J. Downs and E. F. Vogel, "A plant-wide industrial process control problem," Comput. Chem. Eng., vol. 17, no. 3, pp. 245-255, 1993.

[2] T. McAvoy and N. Ye, "Base control for the tennessee eastman problem," Computers \& Chemical Engineering, vol. 18, no. 5, pp. 383-413, 1994.

[3] N. Ricker, "Optimal steady-state operation of the Tennessee-Eastman challenge process," Computers \& Chemical Engineering, vol. 19, no. 9, pp. 949-959, SEP 1995.

[4] - "Decentralized control of the Tennessee Eastman challenge process," J. Process Control, vol. 6, no. 4, pp. 205-221, 1996.

[5] N. Ricker and J. Lee, "Nonlinear model predictive control of the tennessee eastman challenge process," Computers \& Chemical Engineering, vol. 19, no. 9, pp. 961-981, 1995.

[6] Z. Tian and K. A. Hoo, "Multiple model-based control of the tennesseeeastman process," Industrial \& engineering chemistry research, vol. 44 no. 9, pp. 3187-3202, 2005.

[7] T. Larsson, K. Hestetun, E. Hovland, and S. Skogestad, "Self-optimizing control of a large-scale plant: The Tennessee Eastman process," Ind. Eng. Chem. Res., vol. 40, no. 22, pp. 4889-4901, OCT 312001.

[8] S. Skogestad, "Plantwide control: The search for the self-optimizing control structure." J. Process Control, vol. 10, no. 5, pp. 487-507, 2000.

[9] V. Alstad and S. Skogestad, "Null space method for selecting optimal measurement combinations as controlled variables," Ind. Eng. Chem. Res., vol. 46, no. 3, pp. 846-853, 2007.

[10] V. Kariwala, "Optimal measurement combination for local selfoptimizing control," Ind. Eng. Chem. Res., vol. 46, no. 11, pp. 36293634, 2007.

[11] V. Kariwala, Y. Cao, and S. Janardhanan, "Local self-optimizing control with average loss minimization," Ind. Eng. Chem. Res., vol. 47, no. 4, pp. 1150-1158, 2008.

[12] V. Alstad, S. Skogestad, and E. S. Hori, "Optimal measurement combinations as controlled variables," J. Process Control, vol. 19, no. 1, pp. 138-148, 2009.

[13] L. Ye, Y. Cao, Y. Li, and Z. Song, "Approximating necessary conditions of optimality as controlled variables," Industrial \& Engineering Chemistry Research, vol. 52, no. 2, pp. 798-808, 2013.

[14] L. Ye, Y. Cao, and Y. Xiao, "Global approximation of self-optimizing controlled variables with average loss minimization," Ind. Eng. Chem. Res., vol. 54, no. 48, pp. 12040-12 053, 2015.

[15] E. Uerdingen, U. Fischer, R. Gani, and K. Hungerbühler, "A new retrofit design methodology for identifying, developing, and evaluating retrofit projects for cost-efficiency improvements in continuous chemical processes," Industrial \& engineering chemistry research, vol. 44, no. 6 , pp. 1842-1853, 2005. 